\title{
Theoretical Study of Thermodynamic Properties of Liquid Methyl Tert-Butyl Ether (MTBE) Using Ab Initio Calculations and Monte Carlo Simulation
}

\author{
Luciene B. Silva \& Luiz C. G. Freitas
}

\section{Introduction}

Fuel oxygenates are special types of organic compounds blended with gasoline to improve its quality. These compounds raise the oxygen content of gasoline which enhances the combustion process. As a result, the harmful vehicular emissions are reduced.1a MTBE is an ether wherein the oxygen atom is bonded to two alkyl groups (Figure 1). The particular geometry around the oxygen atom and the nature of the $\mathrm{C}-\mathrm{O}$ bond make MTBE a polar molecule. ${ }^{1,2}$ The polarity leads to a high water solubility that combined to MTBE's high vapor pressure and its widespread use in the fuels, causes additional pollution of the urban storm water, the groundwater and the surface water. ${ }^{3}$

Thus in spite of the air quality benefits, there are concerns with the use of MTBE. A growing number of studies have detected MTBE in ground water. In some instances, these contaminated waters are sources of drinking water. Low levels of MTBE can make drinking water supplies undrinkable due to its offensive taste and odor. $1 \mathrm{~b}$ There are opportunities for MTBE to leak into the environment and potentially get in drinking water sources wherever gasoline is stored. There are also opportunities for it to be spilled whenever fuel is transported or transferred. Contamination of drinking water sources can occur from leaking underground and above ground fuel storage tanks, pipelines, refueling spills, automobile accidents damaging the fuel tank, consumer disposal of old gasoline, emissions from older marine engines and to a lesser degree, storm water runoff, and precipitation mixed with MTBE in the air.1c

Several studies have been performed in order to provide developments in technologies for MTBE removal from water. ${ }^{4}$ The gas phase structures and properties of MTBE have been calculated using a variety of ab initio methods. ${ }^{5}$ The structure and energetics of neutral, ionized and protonated MTBE clusters were investigated by vacuum ultraviolet photoionization and ab initio investigations. ${ }^{6}$ Nevertheless, in spite of the great interest in MTBE, the nature and extension of its molecular properties in the condensed phase remain unclear.

Computer simulations methods such as Monte Carlo (MC) are suitable tools for investigations of liquid properties from an atomic level. ${ }^{7,8}$ This methodology provides a direct link between the microscopic details of the system and its macroscopic properties. A key component in simulations is the quality of the force field used to describe the molecular interactions. ${ }^{9}$ Starting from geometries and electrostatic potentials obtained using quantum chemistry methods, force fields for liquid simulations are usually optimized to reproduce experimental pure liquid properties, such as density and heat of vaporization at a given temperature. Typically, intermolecular interactions in force fields are represented by effective pair potentials consisting of Lennard-Jones and Coulomb terms. ${ }^{7}$ Standard Lennard-Jones parameters are available for all common functional groups found in organic liquid. The starting parameters can be further 
optimized to reproduce experimental properties of the liquid phase. The assignment of the partial charges used to describe the coulombic potential is critical in force field development. An approach that has been pursued is to fit the charges to reproduce electronic properties, such as dipole moment and electrostatic potentials. ${ }^{10}$ The most common alternative in liquid simulation has focused on partial charges from fitting to the electrostatic potential surface (EPS) using ab initio calculations. ${ }^{11}$

The present work presents an investigation of the thermodynamic properties of liquid MTBE using Monte Carlo simulation technique. For this purpose a force field has been developed in order to reproduce accurate results for available experimental pure liquid properties.

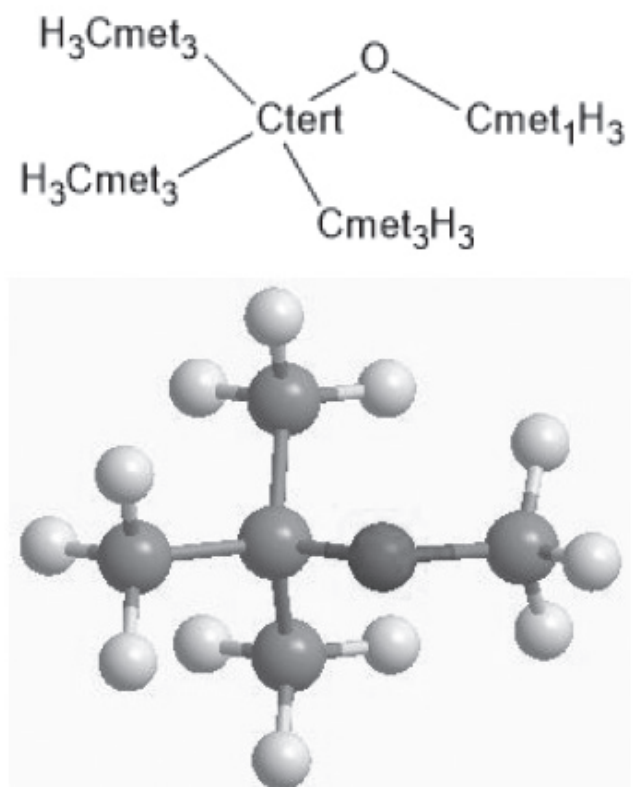

Figure 1: MTBE molecule

$$
E=E_{L J}+E_{\text {Coulomb }}
$$

Where:

$$
\begin{aligned}
& E_{L J}=\sum_{i<j} 4 \epsilon_{i j}\left[\left(\frac{\sigma_{i j}}{r_{i j}}\right)^{12}-\left(\frac{\sigma_{i j}}{r_{i j}}\right)^{6}\right] \\
& E_{\text {Coulomb }}=\sum_{i<j} \frac{q_{i} q_{j}}{r_{i j}}
\end{aligned}
$$

The parametrization of the force field for liquid MTBE was performed as follows: (i) The geometry of the MTBE molecule was obtained using the BP86 functional and Def-Bas6 basis set implemented in the ORCA quantum chemistry program; ${ }^{12}$ (ii) Point charges for each site of the MTBE molecule were calculated using the same functional and basis set above and the CHELPG procedure $^{11}$ in ORCA program; (iii) The Lennard-Jones parameters were empirically optimized from a series of liquid phase simulations in the NPT ensemble at the temperature of $298 \mathrm{~K}$ and pressure of $1 \mathrm{~atm}$; (iv) The geometric parameters of the MTBE monomers were considered rigid through the optimization process.

Monte Carlo simulations using the Metropolis algorithm $^{7}$ were performed with the DIADORIM program. ${ }^{13}$ An initial box containing 500 MTBE monomers was generated and periodic boundary conditions were applied through the simulations.

\section{Results and Discussion}

Table 1 presents the optimized Lennard-Jones parameters and charges for the MTBE liquid simulations. The sites are named according to Figure 1.

Table 1: Lennard-Jones parameters and charges for MTBE simulations

\begin{tabular}{lccc}
\hline \multicolumn{1}{c}{ Site } & $\sigma / \dot{\mathrm{A}}$ & $\varepsilon / \mathrm{kcal} \mathrm{mol}^{-1}$ & $\mathrm{q}$ \\
\hline $\mathrm{O}$ & 2.929 & 0.1260 & -0.48818 \\
Ctert & 3.535 & 0.0594 & 1.05161 \\
Cmet1 & 3.535 & 0.0594 & -0.14319 \\
Cmet3 & 3.535 & 0.0594 & -0.55700 \\
$\mathrm{H}$ & 2.525 & 0.027 & 0.10423 \\
\hline
\end{tabular}


Using the parameters set listed in Table 1, the simulations yielded the thermodynamic properties shown in Table 2. The results were obtained with the MC method in the isotherm-isobaric ensemble with $\mathrm{T}=298$ $\mathrm{K}$ and $\mathrm{p}=1.0 \mathrm{~atm}$, with $0.202 \times 108$ configurations for averaging.

Table 2: Heats of vaporization, $\Delta$ Hvap, density, expansibilities $(\alpha)$ and compressibilities $(k)$ for MTBE liquid simulation

\begin{tabular}{cc}
\hline & This work \\
\hline$\Delta H_{\text {vap }} / \mathrm{kcal} \mathrm{mol}^{-1}$ & $6.739+/-(0.005)^{\mathrm{a}}$ \\
Density $/ \mathrm{g} \mathrm{cm}^{-3}$ & $0.703+/-(.002)^{\mathrm{b}}$ \\
$\alpha / 10^{-5} \mathrm{deg}^{-1}$ & $17.741+/-(8)$ \\
$\kappa / 10^{-6} \mathrm{~atm}^{-1}$ & $20.30+/-(2)$ \\
\hline
\end{tabular}

\section{Conclusion}

Monte Carlo simulations have been used to investigate thermodynamic properties of liquid MTBE. For this purpose, a force field has been developed. An approach of combining charges from fitting to the EPS using the CHELPG procedure and Lennard-Jones parameters empirically optimized provided a proper reproduction of the experimental heat of vaporization and density of the MTBE pure liquid. The force field achieved in this work suggests further studies including the structural characterization of the condensed phase liquid.

\section{Acknowledgments}

The authors acknowledge financial support from FAPESP, CNPq and CAPES.

\section{References}

1. The U.S. Environmental Protection Agency. (a) MTBE in Fuels. Available from: http://www.epa.gov/mtbe/gas.htm; (b) Concerns about MTBE. Available from: http://www.epa.gov/mtbe/water. htm\#concerns; (c) Occurence. Available from: http:/www.epa. gov/mtbe/water.htm\#occurence. 2012. [2013].

2. R. D. Suenram, F. J. Lovas, W. Pereyra, G. T. Fraser and A. R. H. Walker, J. Mol. Spectrosc. 181, 167 (1997).
4. I. Levchuk, A. Bhatnagar and M. Sillanpää, Science of the Total Environment, 415, 476 (2014).

5. L. N. Gregerson, J. S. Siegel and K. K. Baldridge, J. Phys. Chem. A, 104, 11106 (2000).

6. T. M. Di Palma and A. Bende, Chem. Phys. Letters, 18, 561 (2013).

7. M. P. Allen and D. J. Tildesley, Computer Simulations of Liquids, Clarendon Press, Oxford (1987).

8. A. R. Leach, Molecular Modelling: Principles and Applications, Pearson Education (2001).

9. (a) W. F. van Gunsteren and H. J. C. Berendsen, Angew. Chem. Int. Ed. Engl., 29, 992 (1990); (b) W. D. Cornell, P. Cieplak, C. I. Bayly, I. R. Gould, K. M. Merz Jr., D. M. Fergunson, D. C. Spellmeyer, T. Fox, J. W. Caldwell and P. A. Kollman, J. Am. Chem. Soc., 117, 5179 (1995); (c) W. L. Jorgensen, D. S. Maxwell and J. Tirado-Rives, J. Am. Chem. Soc., 118, 11225 (1996).

10. M.M. Francl and L. E. Chirlian, Ver. Comput. Chem., 1, 14 (2001).

11. (a) C. M. Breneman and K. B. Wiberg, J. Comp. Chem. 11, 361, (1990); (b) H. A. Carlson, T. B. Nguyen, M. Orozco and W. L. Jorgensen, J. Comp. Chem. 14, 1240 (1993).

12. F. Neese. The ORCA Program System, Wiley Interdiscip. Rev., Comput. Mol. Sci., 2, 73 (2012).

13. L. C. G. Freitas, J. Braz. Chem. Soc., 20, 1541-1548 (2009).

14. The U.S. Environmental Protection Agency. Available from: http://www.epa.gov/chemfact/s_mtbe.txt. 1994.

\section{Luciene Borges Silva \& Luiz Carlos Gomide Freitas ${ }^{b, *}$}

\footnotetext{
a Departamento de Física, Química e Matemática, Universidade Federal de São Carlos, Campus de Sorocaba, Rod. João Lemes dos Santos, km 110, CEP 18052-780, Sorocaba, São Paulo, Brazil

${ }^{\text {b }}$ Departamento de Química, Universidade Federal de São Carlos, Caixa Postal 676, CEP 13565-950, São Carlos, São Paulo, Brazil

*E-mail: luizcarlosgomidefreitas@gmail.com
}

3. O. C. Braids, Environ. Forensics, 2, 189 (2001). 